\title{
Diagnostic Performance of the Leuko-CRP Index and Procalcitonin in Patients with Suspected Sepsis who Come to the Emergency Room of the IESS Riobamba Hospital in the period July-December 2017
} \author{
Gavilanes Olivo ${ }^{3}$ \\ ${ }^{1}$ Internal Medicine Service, principal investigator, IESS General Hospital, Ecuador \\ ${ }^{2}$ General medicine, collaborator, Health District 11D08, Ecuador \\ ${ }^{3}$ General medicine, collaborator, 06D05 Health District, Ecuador
}

Héctor Fabián Ortega Castillo, ${ }^{1 *}$ Héctor David Tito Tito, ${ }^{2}$ Marco Eduardo Chasipanta Avalos, ${ }^{3}$ Nancy Estefanía

\begin{abstract}
Introduction: Syndrome systemic inflammatory response (SIRS) is activated by the presence of any organic infection to, continuing evolves sepsis, septic shock, multiple organ failure and ends in death.
\end{abstract}

Objective: Determine the utility leuco index/PCR as diagnostic early sepsis.

Materials and Methods: Research has no experimental, of type descriptive, transverse and retrospective during the period July-December 2017, in patients with suspected bacterial sepsis attending IESS Riobamba Hospital emergency. It is utilize for diagnostic validity to the calculation of sensitivity, specificity, positive predictive value (PPV), negative predictive value (NPV), likelihood ratio and ROC curves.

Results: Se and videnctio as Qua r point of co ho rte of the leuco-PCR index a value of619.82 (AUC:0.85; IC-95\%: 0.76-0.95; p<0.05) for a procalcitonin greater than or equal to $1 \mathrm{ng} / \mathrm{dl}$; while for a procalcitonin most or equal to $0.5 \mathrm{ng} / \mathrm{dl}$, an AUC: 0.8 2; CI-95 \%; 0.71-0.9 2; p<0.05 ).

Conclusion: The index leuco-PCR is useful in the diagnosis of sepsis, and alternative accesib him and inexpensive compared to procalcitonin.

Keywords: Leukocytes,CRP, Procalcitonin, Sepsis,SIRS

\section{Introduction}

Sepsis is the clinical response of the systemic inflammatory response syndrome (SIRS), a complex syndrome, product of the activation of the immune system as a result of defense against an infection of any type, its evolution leads to septic shock and subsequent to multi-organ failure (FMO) the same that can end with death, whose outcome can be modified if a timely diagnosis is made. ${ }^{1}$ At present, severe sepsis persists as an important cause of morbidity

and mortality, causing more than $60 \%$ of deaths in patients admitted to the intensive care unit. ${ }^{2}$ Worldwide there are 6 million deaths annually from misdiagnosed sepsis; in Europe it represents 29\% of mortality, in the United States it contributes to $35 \%$ of in-hospital deaths and in Colombia mortality reaches up to $33.6 \%{ }^{3}$ The inflammatory response is important in terms of its pathophysiology, which begins with an identified source of infection that leads to systematic inflammation, known as SIRS, currently considered
Quick Response Code:

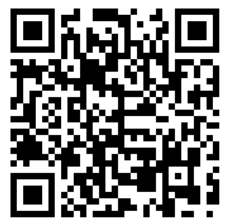

*Corresponding author: Héctor F Ortega, Internal Medicine Service, General Hospital IESS-Riobamba, Avenida Unidad Nacional y Chile, Riobamba, Ecuador

Received: 28 May, 2021

Published: 11 June, 2021

Citation: Héctor FOC, Héctor DTT, Marco ECA, Nancy EGO. Diagnostic Performance of the Leuko-CRP Index and Procalcitonin in Patients with Suspected Sepsis who Come to the Emergency Room of the IESS Riobamba Hospital in the period July-December 2017. Curr Inv Cln Med Res. 2021;1(2):1-4. DOI: 10.53902/CICMR.2021.01.000507 
severe sepsis as a potentially fatal organic dysfunction caused by an abnormal host response to infection, while septic shock refers to sepsis with persistent hypotension. ${ }^{4}$ Endotoxin reproduces many of the characteristics of sepsis when infused in humans, including activation of complement, coagulation, and fibrinolytic systems. These effects can lead to microvascular thrombosis and the production of vasoactive agents; pro-inflammatory mediators in excess: large amounts of pro-inflammatory cytokines released in sepsis patients can leak into the bloodstream, contributing to the progression of a local infection to sepsis, these include tumor necrosis factor alpha (TNFa) and interleukin-1 (IL-1), whose plasma levels s reach a peak early and eventually decrease to ranges undetectable.A cytokines MBAs may cause fever, hypotension, leukocytosis, induction of other proinflammatory cytokines and activation simult to line coagulation and fibrin or lysis. ${ }^{5}$

Relevant bacterial infection biomarkers are presented which are used as a reference,for example hematic biometry, mainly leukocytes and neutrophils; ${ }^{6}$ the desvíor the formal to jointly between these two markers determines the possibility of its origin which can be viral or bacterial and severity d dependent increase or associated complications. C-reactive protein (CRP) is one of the important reactants in the inflammatory response in the acute phase. This increase can be assessed between 12 and 24 hours after the onset of infection, and may remain elevated in the remission phase; however the increase in viral infections is less in comparison bacterial infections.It is used in patients clinically compatible with sepsis and associated with leukocytosis or leukopenia depending on its origin. $^{7}$

Procalcitonin is a biomarker with a sensitivity of $77 \%$ and specificity of $79 \%$, with a positive prediction of $83 \%$, its levels determining the severity of the disease; its increase contributes to a diagnostic and prognostic factor. The serum value of procalcitonin suggests that a value equal to or greater than $0.5 \mathrm{ng} / \mathrm{dl}$ presents a behavior with a rapid elevation in serum in the acute stage of infection and an accelerated decrease shows that the cause is being controlled. ${ }^{8}$ According to what has been described, due to the variation in the markers already mentioned according to each phase of the septic process, an index that includes leukocytes and CRP vs procalcitonin is proposed in order to predict early bacterial sepsis of any origin in patients attending the emergency. Also taking into consideration these aspectsof accessibility, low cost and speed in the results of these biomarkers.

\section{Meall}

It is a study not experimental, of descriptive, cross-sectional and retrospective. Serecopilaron data from medical records that were found in the system computer AS400, complying with the rules of confidentiality of information, consent and authorization of coordination of teaching and research of the General Riobamba IESS hospital. S and analyzed 240 records, of which met the inclusion criteria 61. The latter were: age over 18 years, suspicion or confirmation of bacterial sepsis of any origin in patients admitted for emergency, absence of renal failure, liver failure, myeloproliferative disorders, previous malignancy or other recently identified hematological disorders,absence of gout or other rheumatic disease of autoimmune etiology and non-use of corticosteroids.It was also taken into account for the inclusion of subjects in the study availability Form buffy, PCR and P rocalcitonina, with results within the first 24 hours. The following parameters were also taken into account at hospital admission: temperature, heart rate, respiratory rate, oxygen saturation, systolic blood pressure, Glasgow coma scale. With them, SIRS and q-SOFA scales were applied to determine suspected sepsis.All data were entered into the SPSS version 23.0 statistical package, where a descriptive and inferential analysis was performed. The diagnostic validity of the index leuco-PCR compared to procalcitonin was determined through the calculation of sensitivity, specificity, positive predictive value (PPV), negative predictive value (NPV), positive likelihood ratio ( $\mathrm{LR}+$ ) and negative (LR-), ROC curves to establish the best cohort point and the respective analysis of the area under the curve (AUC). S and worked co $n$ one level of confidence of $95 \%$ and an alpha of 0.05 .

\section{Results}

Of the study population ( $\mathrm{n}=61), 31(50.8 \%)$ corresponded to the male gender and $30(49.2 \%)$ to the female gender, with an average age of 72 years, standard deviation of 18.07, a minimum of 31 and a maximum of 94 years, instead of residence found to be 32(52.5\%), subjects of the urban area and 29(47.5\%) of the rural area. The main infectious diseases found were: Respiratory or s $47.5 \%(29)$, gastrointestinal $29.5 \%(18)$, it is not identified or $11.5 \%(7)$, soft tissue infections $8.2 \%(5)$ and urinary 3.3\%(2). On the other hand, the main comorbidities identified were: cardiovascular $26.2 \%(16)$, chronic respiratory $11.5 \%(7)$, endocrine $9.8 \%(6)$, musculoskeletal $4.9 \%(3)$, renal is $4.9 \%(3)$, oncological $3.3(2)$ and neurological $1.6 \%(1)$. Other results were: value of lecocytes with a mean of 11.410 .9 cells $/ \mathrm{mm}^{3}$; with a standard deviation of 6.424 .17 , minimum value of 1.800 and maximum value of 30.800 cells $/ \mathrm{mm}^{3}$; $\mathrm{t}$ average temperature $37.20^{\circ} \mathrm{C}$, standard deviation 0.84 , Minimum value or 36.00 , maximum value $39.50^{\circ} \mathrm{C}$; frequency card average 81.62 beats/min, with a standard deviation of 18.66, minimum value 18 and maximum value 130 beats/min; mean respiratory rate of 19.39 breaths/min, standard deviation 3.68, minimum value 12 and maximum value of 32 breaths/min; oxygen saturation with mean of $87.10 \%$; standard deviation 6.56; minimum value 69 and maximum value of $99 \%$; mean systolic pressure of $116.41 \mathrm{mmhg}$, standard deviation of 25.70; minimum value 55 and maximum value $170 \mathrm{mmhg}$; Glasgow scale with mean of 13.92; with standard deviation 2.11,minimum value 5 and maximum value of 15 .

The biomarker results were as follows: mean CRP of $98.60 \mathrm{mg} /$ dl; standard deviation of 130.30, minimum value 1.32 and maximum value $768 \mathrm{mg} / \mathrm{dl}$; mean procalcitonin of $7.41 \mathrm{ng} / \mathrm{dl}$; standard deviation 17.28; minimum value 0.02 and maximum value $92.50 \mathrm{ng} / \mathrm{dl}$; mean leuko index/CRP of 1.287.62; standard deviation of 2.486.41; minimum value 4.80 and maximum value 15.360 (Тable 1). In the inferential analysis, a value of 619 was evidenced as the best cohort point of the leuko-CRP index.82 (AUC: 0.85 CI-95\% $0.76-0.95 ; \mathrm{p}<0.05$ ) for greater or equal procalcitonin $1 \mathrm{ng} / \mathrm{dl}$, with a sensitivity of $80 \%$, specificity $72 \%$, positive predictive value (PPV) 
$66 \%$, negative predictive value (NPV) $83 \%$, positive likelihood ratio positive post-test probability $66.5 \%$, negative post-test probability 2.88, negative view ratio or similarity 0.28 , prevalence rate $41 \%, \quad 16.2 \%$, diagnostic odds ratio 10.40 (Table 2) (Graph 1 ).

Table 1: Descriptive statistics of the quantitative variables in the study population.

\begin{tabular}{|c|c|c|c|c|c|}
\hline & \multirow{2}{*}{ Half } & \multirow{2}{*}{ Standard Deviation } & \multirow{2}{*}{ Standard Error } & \multicolumn{2}{|c|}{ 95\% Confidence Interval } \\
\hline & & & & Lower & Higher \\
\hline Age & 72.59 & 18.06 & 1.49 & 14.72 & 20.72 \\
\hline Leukocytes & $11,410.90$ & $6,424,177$ & 689,729 & $4,933,520$ & $7.648,618$ \\
\hline PCR & 98.6044 & 130.3 & 29.58401 & 73.06837 & 185.2654 \\
\hline Leuco-CRP index & $1.287,624$ & 2.486 .4154 & $7,252,327$ & 916.4777 & $3.734,1894$ \\
\hline Procalcitonin & 7.4144 & 17.27772 & 3.81509 & 8.55596 & 23.76296 \\
\hline Temperature & 37.197 & 0.8460 & 0.896 & 0.6514 & 0.9995 \\
\hline Heart rate & 81.62 & 18.665 & 2.356 & 13.973 & 23.194 \\
\hline Respiratory rate & 19.39 & 3.675 & 0.480 & 2.618 & 4.536 \\
\hline Oxygen saturation & 87.10 & 6.564 & 0.681 & 5.153 & 7.822 \\
\hline Systolic pressure & 116.41 & 25.700 & 2.022 & 21.372 & 29.332 \\
\hline Glasgow & 13.92 & 2.108 & 0.360 & 1.356 & 2.750 \\
\hline
\end{tabular}

Source: database 23.0

Table 2: Test result variable (s): LEUCO INDEX - CRP.

\begin{tabular}{|c|c|c|c|c|}
\hline \multirow{2}{*}{ Area } & \multirow{2}{*}{ Standard error } & \multirow{2}{*}{ Asymptotic significance } & \multicolumn{2}{|c|}{ 95\% asymptotic confidence interval } \\
\cline { 3 - 5 } & & 0.047 & Lower limit & Upper limit \\
\hline 856 & 0.764 &, 948 \\
\hline
\end{tabular}

Source: database 23.0

a. Under the nonparametric assumption

b. Null hypothesis: true area $=0.5$

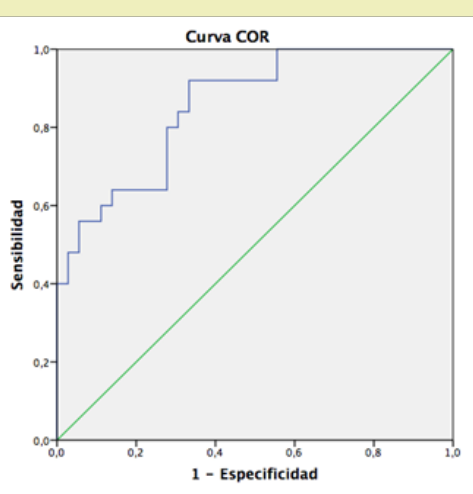

Graph 1: Curve operant index leuco receptor/PCR vs. greater than or equal procalcitonin $1 \mathrm{ng} / \mathrm{dl}$, in bacterial sepsis. Area down the curve

On the other hand, for a procalcitonin greater than or equal to 0.5ng/dl, an AUC: 0.82; CI-95\%; 0.71-0.92; p<0.05; sensitivity 72\%, specificity 76\%, PPV 77\%, NPV 71\%, positive likelihood ratio 2.98 ,

Table 3: Test result variable (s): INDICE_LEUCO_PCR.

\begin{tabular}{|c|c|c|c|c|}
\hline \multirow{2}{*}{ Area } & \multirow{2}{*}{ Standard error } & \multirow{2}{*}{ Asymptotic significance } & \multicolumn{2}{|c|}{$95 \%$ asymptotic confidence interval } \\
\hline & & & Lower limit & Upper limit \\
\hline 816 & ,053 & 000 & ,712 & ,919 \\
\hline
\end{tabular}

\section{Source: database 23.0}

a. Under the nonparametric assumption

b. Null hypothesis: true area $=0.5$ 


\section{Discussion}

The results are intended to provide a simplified model for predicting cases of Sep sis in patients admitted to the emergency unit by an infectious process. The index includes basic leuco PCR studies readily available biomarkers in the early is hospital substance in suspected cases or high probability Sep sis, therefore; represents a useful tool to optimize both empirical and specific treatments early to reduce the mortality rate. ${ }^{5}$ According to the results of our study, factors significantly related to the possible development of sepsis, including age, can be confirmed after the initial assessment, finding patients with an average age of 72 years, a minimum of 31 and a maximum of 94 years, a value that is higher than the study by Agusntín Julián Jiménez et al. ${ }^{1}$ In which the ages oscillate between 52 years with a range of 18 and 98 years, an important risk factor due to the high incidence of infectious processes in the elderly and the presence of comorbidities, being more frequent the probability of the development of sepsis whose late diagnosis decreases life expectancy.

Sadaf Zafar, at the Toledo University Hospital Complex (Spain). ${ }^{2}$ He still mentions Shapiro's proposal, which represented one of the most important references in the emergency department, this model that classifies the risk of bacteraemia as low $(<1 \%)$, moderate (7-9\%) and high (15-26\%) based on major and minor criteria, including leukocytes $>18,000 / \mathrm{mm}^{3}$. Agustín Julián Jiménez in 2019 , ${ }^{3}$ establishes a leukocyte count $>12,000 / \mathrm{mm}^{3}$ as a predictor of sepsis, a value somewhat higher than our data, taking into account that according to the results of our study, leukocytes greater than $11.4 \mathrm{X} 10^{3} / \mathrm{mm}^{3}$ it is also considered indicative of sepsis. According to the study of Ricardo Rubio y Esperanza Lepe, at the University Hospital of Valme (Spain). ${ }^{9-15}$ It was determined that the CRP of $20 \mathrm{mg} / \mathrm{L}$, equivalent to $200 \mathrm{mg} / \mathrm{dl}$ plus procalcitonin greater than $5 \mathrm{mg} / \mathrm{L}$ are indicative of sepsis. In comparison with our study, CRP determined the evolution to sepsis by having avalue greater than $1.32 \mathrm{mg} / \mathrm{dl}$.

Regarding the study by Laura Magrini et al. In a retrospective observational study carried out in a S. Andrea hospital in Rome in the emergency department, 263 male and 250 female subjects were studied with a mean age of 71.7, in our study the study population was 31 male and female subjects 36 with a mean age of 72 . In the analysis of the ROC curves for the diagnosis of sepsis the PCT obtained a mean 17.16, AUC: $0.79, \mathrm{P}<0.0001$ in the HR a mean 102.25 beats/min, temperature $37.58^{\circ} \mathrm{C}$; CRP AUC: $0.72 \mathrm{p}<0.001,95 \% \mathrm{CI}$; In our study, the ROC curve of PCT obtained a mean 17.27, AUC: 0.82, $\mathrm{p}<0.05,95 \% \mathrm{CI}$,in HR 81.62 beats/min, temperature 37.19. Based on the study of Alberto Redondo-González; With a total of 349 patients where the main causes of sepsis were intrahospital infections, several prognostic sepsis scales were also associated,as part of the assessment the Glasgow scale was less than 14 and the $\mathrm{ABC}$ index 0.78 with a $95 \% \mathrm{CI} ; 0.72-0.84, \mathrm{p}<0.001$; while our study presents a Glasgow scale $<14$ (13.92), which maintains the similarity that in cases of sepsis, inflammatory factors do present high degrees of neurological alteration.At the time of the new updates that have been raised based on new sepsis criteria, we must consider the mean arterial pressure as a point of greatest importance as indicated by Gómez and Gómez when defining guidelines on the changes produced by sepsis, as well; Within our study, systolic values greater than $116 \mathrm{mmHg}$ are defined, with which; presenting lower systolic pressures, we found a high probability of sepsis or septic shock. . $^{16,17,18}$

\section{Acknowledgments}

None.

\section{Funding}

None.

\section{Conflicts of Interest}

The authors do not refer any type of interest.

\section{References}

1. Gregory A. Schmidt M, Jess Mandel M. Evaluation and management of suspected sepsis and septic shock in adults. UpToDate. 2018;1-25.

2. Amos Etzioni M. Leukocyte-endothelial adhesion in the pathogenesis of inflammation. UpToDate. 2018;1-9.

3. Fernando A, Beatriz S. Management of the septic patient in the emergency room. Medical Watchtower. 2017;11:7-16.

4. Irving Kushner M. Acute phase reactants. UpToDate. 2017;1-16.

5. Angel Vega ACea. Procalcitonin as diagnostic biomarker of parapneumonic pleural effusion or empyema. Thoracic Surgery (NTC). 2017;76 (1):7-13.

6. Chanu R, Michael KM. Procalcitonin use lower respiratory tract infections. UpToDate. 2020;1(1):1-27.

7. Alfredo Prado MC. Molecular markers in the diagnosis and prognosis of sepsis, sepsis severe and septic choke. Sys Rev Pharm. 2016;1(65):145-155.

8. Konrad R. Misdiagnosed sepsis is now a world health priority for WHO. Global sepsis alliance. 2017;1(1):1-2.

9. Aviles García M. Bacteremia, sepsis and septic shock. Intesiva Medicine Service. 2018;12(52):3066-3075.

10. Remi N. Sepsis syndromes in adults: Epidemiology, definitions, clinical presentation, diagnosis, and prognosis. Uptodate. 2020;1(1):1-35.

11. Michael JS. Sepsis. Emergency Medicine. 2019.

12. Remi N. Pathophysiology of sepsis. UpToDate. 2020;1(1):1-26.

13. Prangsai W. Bodin K. Comparison the accuracy of early warning scores with sSOFA and S IRS for predicting sepsis in the emergency department. ClinicalKey. 2020;1(1):1-14.

14. Suberviola B, Castellanos A, González A, et al. Prognostic value of procalcitonin clearance CRP and leukocytes in septic shock. Med Intensive. 2012;36(3):177-184.

15. Ricardo R. Esperanza L. Management of requests for procalcitonin in the Biochemistry emergency laboratory. Eur J Health Research. 2019;5(2):177-184.

16. Agustin J. Francisco G. Usefulness of biomarkers to predict bacteremia in patients with infection in the emergency room. Revision. 2017;30(4):245-256.

17. Redondo-González A, Varela-Patiño M, Álvarez-Manzanares J, et al. Assessment of severity scales in sepsis patients in a code in a hospital emergency department. Rev Esp Quimioter. 2018;31(4):316-322.

18. Gomez-Gomez,Sanchez Luna JP, Perez Beltran CF, et al. Septic shock. What we knew and what we should know. Med Interna. 2017;36(3):177-184. 